m/s

médecine/sciences $1998 ; 14: 845-7$

\title{
OBÉSITÉ : GRANDEUR ET SERVITUDE DES MODĖLES ANIMAUX
}

e gène agouti est le premier d'une série de cinq gènes, clonés entre 1992 et 1997, dont les mutations ont été incriminées dans divers syndromes d'obésité chez les rongeurs. Parmi ceux-ci, le très médiatique gène $o b$ codant pour la leptine et d'autres moins célèbres comme le gène fat et le gène tub (Tableau I). Ces découvertes ont été réalisées grâce à l'amélioration des techniques de clonage positionnel mais aussi grâce aux investissements réalisés outre-Atlantique par plusieurs compagnies pharmaceutiques. Ces investissements sont-ils «rentables "? En d'autres termes, le clonage de ces gènes soulève-t-il l'espoir d'une thérapeutique de l'obésité ? L'histoire exemplaire de la leptine, dont les lecteurs de médecine/sciences ont été largement informés, est la démonstration même que cet espoir est encore bien lointain... sauf pour les cinq patients connus à ce jour qui présentent un
M. Guerre-Millo : directeur de recherche à l'Inserm. Inserm U. 465, Institut biomédical des Cordeliers, 15, rue de l'École-de-Médecine, 75006 Paris, France.

$m / s n^{\circ} 8-9$, vol. 14, août-septembre 98
Tableau I

\section{OBÉSITÉS MONOGÉNIQUES CHEZ LES RONGEURS ET CHEZ L'HOMME}

\begin{tabular}{|c|c|c|c|c|}
\hline OBÉSITÉS & MONOGÉ & NIQUES & $\begin{array}{c}\text { Tableau I } \\
\text { CHEZ LES RC }\end{array}$ & ONGEURS ET CHEZ L'HOMME \\
\hline Protéine & Mutation & Espèce & Références & Anomalie \\
\hline Agouti & $A^{y}, A^{v y}$ & souris & {$[1,2]$} & $\begin{array}{l}\text { Antagonisme du récepteur } \\
\text { mélanocortinergique MC4-R }\end{array}$ \\
\hline Leptine & $\begin{array}{l}o b \\
o b^{2 J} \\
- \\
-\end{array}$ & $\begin{array}{l}\text { souris } \\
\text { souris } \\
\text { homme } \\
\text { homme }\end{array}$ & $\begin{array}{c}{[3]} \\
{[15]} \\
{[7]} \\
{[8]}\end{array}$ & Absence de leptine \\
\hline $\begin{array}{l}\text { Récepteur } \\
\text { de la } \\
\text { leptine }\end{array}$ & $\begin{array}{l}d b \\
d b^{3 J} \\
f a \\
f a^{k} / f a^{c p} \\
-\end{array}$ & $\begin{array}{l}\text { souris } \\
\text { souris } \\
\text { rat } \\
\text { rat } \\
\text { homme }\end{array}$ & $\begin{array}{c}{[11,12]} \\
{[16]} \\
{[17,19]} \\
{[12,20,21]} \\
{[9]}\end{array}$ & $\begin{array}{l}\text { Absence de transmission } \\
\text { signal leptinique }\end{array}$ \\
\hline $\begin{array}{l}\mathrm{CPE}^{*} \\
\mathrm{PC}^{*}{ }^{*}\end{array}$ & $\begin{array}{l}\text { fat } \\
-\end{array}$ & $\begin{array}{l}\text { souris } \\
\text { homme }\end{array}$ & $\begin{array}{c}{[4]} \\
{[22]}\end{array}$ & $\begin{array}{l}\text { Défaut de maturation de } \\
\text { propeptides et de } \\
\text { prohormones }\end{array}$ \\
\hline POMC*** & - & homme & [10] & $\begin{array}{l}\text { Absence de peptides dérivés } \\
\text { de la POMC }\end{array}$ \\
\hline tub & tubby & souris & {$[5,6]$} & $?$ \\
\hline
\end{tabular}

* CPE : carboxypeptidase E; ** PC1 : proconvertase $1, * * *$ POMC : proopiomélanocortine. Nous remercions Naima Moustaid Moussa pour sa participation à l'élaboration de ce tableau. 
déficit en leptine (protéine ou récepteur) $[7,8]$ ! Néanmoins, les modèles animaux d'obésité ont été source de progrès considérables dans la compréhension des mécanismes complexes qui règlent l'homéostasie énergétique.

Pendant plus de 30 ans, les caractéristiques phénotypiques des rongeurs génétiquement obèses ont été analysées sans préjuger de la fonction de la protéine déficiente. Aujourd'hui, le nouveau défi consiste à comprendre comment la perte ou le gain de fonction d'une protéine unique produit les défauts observés et conduit au surdéveloppement de la masse adipeuse. Pour réussir dans cette étape, il faut tout d'abord découvrir la fonction de la protéine invalidée. Ce n'est pas toujours possible : le gène tub code pour une protéine dont la fonction exacte reste inconnue à ce jour $(m / s$ 1996, no 8-9, p. 974) [5, 6]. Au contraire, on a parfois la "chance " de trouver une mutation dans un gène connu. C'est le cas de la mutation $f a t$, qui affecte le gène de la carboxypeptidase $\mathrm{E}\left(\mathrm{m} / \mathrm{s} 1995, n^{\circ} 8\right.$, p. 1171) [4]. On reste cependant sur sa faim : comment un défaut de maturation protéique engendre-t-il l'obésité d'émergence tardive qui caractérise les mutants fat/fat ? La découverte d'une invalidation $\mathrm{du}$ gène de la proconvertase 1 chez une patiente obèse $\left(\mathrm{m} / \mathrm{s} 1997, n^{\circ} 12\right.$, p. 1448) [22] confirme l'implication des processus de maturation enzymatique, sans éclaircir le mystère des mécanismes moléculaires produisant l'obésité ! Il est tentant de mettre en cause un défaut de maturation de la proopiomélanocortine (POMC), dont dérivent plusieurs peptides, comme les endorphines et la mélanocortine, qui modifient la prise alimentaire chez les rongeurs. La découverte récente de mutations dans le gène de la POMC engendrant une obésité sévère et précoce chez deux jeunes patients non apparentés, est en faveur de cette hypothèse ( $m / s$ 1998, $n^{\circ} 8-9, p$. 955) [10]. Le rôle inattendu de la mélanocortine dans l'homéostasie pondérale a été découvert grâce au clonage du gène agouti, dont le produit s'est révélé être un puissant antagoniste de cette hormone $[1,2]$. Par la suite, l'invalidation du gène du récepteur MC4-R chez la souris a parfaitement confirmé l'importance des circuits mélanocortinergiques (voir l'article de Naima Moustaid Moussa, p. 898 de ce numé$r o$ ). Enfin, en ce qui concerne la leptine et ses récepteurs, ce sont à la fois des recherches d'analogie structurale et des études fonctionnelles qui ont permis de découvrir le rôle biologique de l'hormone, concrétisant ainsi le concept de «lipostat » qui reposait sur les études phénotypiques des souris $o b / o b$ et $d b / d b$. En fait, le clonage du gène a permis de découvrir que la leptine outrepasse largement ce rôle. En particulier, l'importance de l'hormone pour la fonction de reproduction est maintenant bien établie, y compris dans l'espèce humaine [8, 9]. Il faut néanmoins garder à l'esprit qu'aux niveaux cellulaire et moléculaire, son mode d'action précis est loin d'être décrypté.

Malheureusement, il est clair que les modèles animaux d'obésité monogénique ne sont représentatifs que d'une infime minorité de cas chez l'homme. Certainement plus proches de l'obésité humaine, des modèles animaux d'obésité polygénique ont commencé à être étudiés dans les années 1990. Ces modèles sont obtenus par croisement entre souches de souris très dissemblables pour le pourcentage de masse grasse ou la sensibilité à l'obésité nutritionnelle, par exemple. Ils permettent des études génétiques poussées qui aboutissent à associer certaines régions chromosomiques aux caractéristiques d'intérêt. Cependant, un long chemin reste à parcourir pour retrouver les gènes localisés dans ces régions et comprendre leur rôle respectif dans l'homéostasie énergétique.

L'exemple du gène de la protéine découplante UCP2 (m/s 1997, $n^{\circ} 4$, p. 607), cartographié dans une région du chromosome 7 murin clairement associée à l'obésité dans plusieurs modèles polygéniques démontre la puissance de cette approche qui devrait permettre, dans un avenir proche, de découvrir de nouveaux gènes candidats, connus ou inconnus. Il existe de multiples façons de provoquer une obésité chez l'animal, mais cela arrive parfois d'une manière tout à fait inattendue. Le dévelop- pement d'un phénotype obèse fait partie des surprises que réservent les invalidations de gène! Un sous-type de récepteur de la bombésine $(\mathrm{m} / \mathrm{s}$ 1998, $n^{\circ} 2$, p. 223), des métallothionines et même certains récepteurs d'adhérence leucocytaires sont autant d'exemples de protéines codées par des gènes dont l'invalidation provoque une obésité plus ou moins prononcée. Comment des protéines aussi différentes, ayant des fonctions connues sans rapport apparent avec l'homéostasie énergétique, peuvent-elles être impliquées dans le développement d'une obésité ? Cela reste un mystère complet à ce jour. A l'inverse, l'invalidation de certains gènes que l'on pensait très impliqués dans le contrôle du poids corporel, n'a pas provoqué le phénotype escompté. C'est le cas, par exemple, des souris déficientes en neuropeptide $\mathrm{Y}$ ou en récepteur $\beta 3$-adrénergique $[13,14]$. Ces modèles soulèvent plusieurs questions, en particulier, celle de l'influence du patrimoine génétique et de l'apport calorique dans l'expression du phénotype. Il est possible que l'invalidation de ces mêmes gènes dans un environnement génétique ou nutritionnel différent puisse conduire à l'obésité. Il ne faut pas oublier que c'est aussi grâce aux modèles animaux que l'importance de ces facteurs a été reconnue de longue date. Les régimes enrichis en graisse et les régimes «cafétéria » sont générateurs de prise de poids excessive chez les rongeurs et, tout comme chez l'homme, certaines souches sont plus sensibles que d'autres à la suralimentation!

Les neurophysiologistes m'en voudraient de ne pas citer les modèles lésionnels d'obésité. Par exemple, les modèles de rongeurs lésés au niveau de l'hypothalamus ventro-médian trouvent leur contrepartie en pathologie humaine chez les patients atteints de tumeurs hypothalamiques ou présentant des séquelles opératoires de craniopharyngiome, souvent associées à une hyperphagie majeure. Ces modèles ont été d'une importance capitale dans la caractérisation des régions cérébrales impliquées dans l'homéostasie énergétique. Ils ont ouvert la voie aux recherches 
actuelles qui, à l'aide des nouveaux outils moléculaires disponibles, visent à décrypter la complexité des circuits peptidergiques qui contrôlent la prise alimentaire et la dépense énergétique (voir l'article de Denis Richard, p. 877 de ce numéro).

La diversité des phénotypes d'obésité et la multiplicité de leurs causes aussi bien chez l'animal que chez l'homme, suggèrent que des circuits parallèles et non en série sont impliqués, avec probablement des réseaux redondants. Merci à nos prédécesseurs qui surent conserver diverses souches de rongeurs obèses, ce qui a permis 30 à 40 ans plus tard de comprendre un peu mieux certains des mécanismes de contrôle de l'homéostasie pondérale. Il est clair que les modèles animaux existants et futurs n'ont pas livré tous leurs secrets. Espérons qu'ils contribueront à la découverte d'un moyen d'enrayer le fléau grandissant de l'obésité

\section{RÉFÉRENCES}

1. Bultman SJ, Michaud EJ, Woychik. Molecular characterization of the mouse agouti locus. Cell 1992 ; 71 : 1195-204.

2. Miller MW, Duhl DMJ, Vrieling H, Cordes SP, Ollmann MM, Winkes BM, Barsh GS. Cloning of the mouse agouti gene predicts a secreted protein ubiquitously expressed in mice carrying the lethal yellow mutation. Genes Dev 1993 ; 7 : 454-67.

3. Zhang Y, Proenca R, Maffei M, Barone M, Leopold L, Friedman JM. Positional cloning of the mouse obese gene and its human homologue. Nature 1994 ; 372 : 425-32.

4. Naggert JK, Fricker LD, Varlamov O, Nishina PM, Rouille Y, Steiner DF, Carroll RJ, Paigen BJ, Leiter EH. Hyperproinsulinemia in obese fat/fat mice associated with a car- boxypeptidase E mutation which reduces enzyme activity. Nat Genet 1995 ; 10 : 135-42.

5. Noben-Trauch K, Naggert JK, North MA, Nishina PM. A candidate gene for the mouse mutation tubby. Nature 1996 ; 380 : 534-8.

6. Kleyn PW, Fan W, Kovats SG, Lee JJ, Pulido JC, et al. Identification and characterization of the mouse obesity gene tubby: a member of a novel gene family. Cell 1996 ; 85 : 281-90.

7. Montague CT, Faroogi IS, Whitehead JP, Soos MA, Rau H, et al. Congenital leptin deficiency is associated with severe early-onset obesity in humans. Nature 1997 ; 387 : 903-8.

8. Strobel A, Issad T, Camoin L, Ozata M, Strosberg AD. A leptin missense mutation associated with hypogonadism and morbid obesity. Nat Genet 1998; 18 : 213-5.

9. Clément K, Vaisse C, Lahlou N, Cabrol S, Pelloux V, et al. A mutation in the human leptin receptor gene causes obesity and pituitary dysfunction. Nature 1998 ; 392 : 398-401.

10. Krude H, Biebermann H, Luck W, Horn R, Braban G, Grüters A. Severe early-onset obesity, adrenal insufficiency and red hair pigmentation caused by POMC mutations in humans. Nat Genet 1998; 19 : 155-7.

11. Chen H, Charlat O, Tartaglia LA, Woolf $\mathrm{EA}$, Weng $\mathrm{X}$, et al. Evidence that the diabetes gene encodes the leptin receptor : identification of a mutation in the leptin receptor gene in $d b / d b$ mice. Cell $1996 ; 84: 491-5$.

12. Lee GH, Proenca R, Montez JM, Carroll KM, Darvishzadeh JG, Lee JI, Fiedman JM. Abnormal splicing of the leptin receptor in diabetic mice. Nature 1996 ; 379 : 632-5.

13. Erickson JC, Clegg KE, Palmiter RD. Sensitivity to leptin and susceptibility to seizures of mice lacking neuropeptide Y. Nature $1996 ; 381: 415-21$.

14. Dulloo A, Seydoux J, Giacobino JP, Huarte J, Ody C. Targeted gene disruption reveals a leptin-independent role for the mouse beta3-adrenoceptor in the regulation of body composition. I Clin Invest $1997 ; 100$ : 1098-106.
15. Moon BC, Friedman JM. The molecular basis of the obese mutation in $o b^{2 j}$ mice. $G e-$ nomics 1997, 42 : 155-6.

16. Lee GH, Li C, Montez J, Halaas J, Darvishzadeh J, Friedman JM. Leptin receptor mutations in $129 \mathrm{db}^{3 \mathrm{~J}} / \mathrm{db}^{3 \mathrm{~J}}$ mice and NIH $\mathrm{fa}^{\mathrm{cp}} / \mathrm{fa}^{\mathrm{cp}}$ rats. Mamm Genome 1997 ; 8 : 445-7.

17. Phillips MS, Liu O, Hammond HA, Dugan V, Hey PJ, Caskey CT, Hess JF. Leptin receptor missense mutation in the fatty Zucker rat. Nat Genet 1996 ; 13 : 18-9.

18. Chua SC, White DW, Wu-Peng XS, Liu $\mathrm{SM}$, Okada N, et al. Phenotype of fatty due to Gln269Pro mutation in the leptin receptor (Lepr). Diabetes 1996 ; 45 : 1141-3.

19. Iida M, Murakami T, Ishida K, Mizuno A, Kuwujima M, Shima K. Phenotype-linked aminoacid alteration in leptin receptor cDNA from Zucker fatty (fa/fa) rat. Biochem Biophys Res Commun 1996 ; 222 : 19-26.

20. Takaya K, Ogawa Y, Hiraoka J, Hosoda K, Yamori Y, Nakao K, Koletsky RJ. Nonsense mutation of leptin receptor in the obese spontaneously hypertensive Koletsky rat. Nat Genet 1996 ; 14 : 130-1.

21. Wu-Peng XS, Chua Jr SC, Okada N, Liu SM, Nicolson M, Liebel RL. Phenotype of the obese Koletsky (f) rat due to Tyr763Stop mutation in the extracellular domain of the leptin receptor (Lepr). Evidence for deficient plasma-to-CSF transport of leptin in both Zucker and Koletsky obese rat. Diabetes $1997 ; 46: 513-8$

22. Jackson RS, Creemers JWM, Ohagi S, Raffin-Sanson ML, Sanders L, Montague CT, Hutton JC, O'Rahilly S. Obesity and impaired prohormone processing associated with $\mathrm{mu}-$ tations in the human prohormone convertase 1 gene. Nat Genet 1997 ; 16 : 303-6.

\section{CONFÉRENCES JACQUES MONOD 1998}

\section{PLASTICITÉ SYNAPTIQUE, DYNAMIQUE DES ASSEMBLÉES NEURONALES ET FLEXIBILITÉ DES REPRESENTATIONS COGNITIVES \\ AUSSOIS (France) - 30 novembre - 4 décembre 1998 \\ Président: $\quad$ FREGNAC Yves}

CNRS, Institut Alfred-Fessard, Avenue de la Terrasse, F-91 198 Gif-sur-Yvette Cedex, France.

Phone - Téléphone : + 33169823415 - Fax - Télécopie : + 331698234 27. E-mail - Courrier électronique : fregnac @iaf.cnrs-gif.fr

Conférenciers : Bachevalier J., Bear M., Berthoz A., Bliss T., Changeux J.-P., Collingridge G., Crepel F., Dehaene S., De Schonen S., Doupe A., Edeline J.-M., Fox K., Frackowiak R., Frégnac Y., Gervais R., Gilbert C., Kew J., Konnerth A., Laroche S., Le Masson G., Markram H., Masson C., O'Keefe J., Polk T., Ramachandran V., Recanzone G., Rolls E., Sagi D., Salin P., Savage-Rumbaugh E.-S., Schultz W., Tanaka K., Vaadia E., Wilson M. 